\title{
Specialist clinics: a better way to care?
}

\author{
W J Mutch
}

\begin{abstract}
A questionnaire on the development of specialist clinics was distributed to the members of the Association of British Neurologists and completed by $104 \mathrm{mem}$ bers ( 85 consultants). Half of the respondents already ran a specialist clinic. The same proportion felt that a Parkinsonism clinic would be useful despite the fact that only $20 \%$ stated that they would run one. It is argued that specialist clinics facilitate the effective management of conditions such as Parkinson's disease, especially if supported by a specialist nurse or other appropriate staff.
\end{abstract}

In recent years there has been a growing interest in the delivery of health care, driven in part by medical audit but also by the White Paper "Working for Patients".'

My contribution has nothing profound to offer in the philosophy of managing patients with chronic neurological conditions, rather it examines various facets of a possible package of measures which can respond flexibly and effectively to patients' needs, whilst they continue to live in the community. It does not deal with respite and terminal care which are an important part of any comprehensive package of care. The management of Parkinsonism is used as an example since it is common and reflects many of the situations which may arise during the course of a chronic neurological condition.

\section{SPECIALIST CLINICS}

Specialist clinics are not new to neurology. In a report "Standards of Care for Patients with Neurological Disease" a working group of the Royal College of Physicians, composed principally of neurologists, recommended that they should be evaluated. ${ }^{2}$

Why might such clinics be appropriate for the management of Parkinsonism? In community terms, it is a common condition but each general practitioner, assuming both an average practice size and age distribution within that practice will have only four to five patients with the disease. This is in reality too few patients to develop adequate expertise given the range of problems likely to be encountered such as the difficulties with diagnosis; the wide range of clinical problems which patients experience both by day $^{3}$ and night; ${ }^{4}$ the troublesome side effects of drugs; the many elderly patients with other pathologies requiring further appropriate medication and the resulting potential for drug interaction; the major problems in dealing with later stage motor phenomena and the additional burden of complex psychiatric problems. In addition, general practitioners often do not have access to therapy services, which are essential for effective management.

The advantages of a clinic include confirmation of the diagnosis by a specialist and recruitment to early stage therapy trials. A greater concentration of experience gives better management of simple problems such as constipation, which are unfortunately often poorly dealt with, and also of more complex problems such as psychiatric manifestations and on/off phenomena. It also gives access to a full multidisciplinary team, can lead to more effective use of resources (drugs, services or people), and can act as an advice/education centre both on a drop-in and telephone basis. In addition it offers an identified source of excellence for patients, carers and for professionals, who can use it to benefit their education either through informal attendance or as part of a training rotation. Finally it is invaluable for a wide range of research issues.

A further perspective comes from the NHS White Paper "Working for Patients", ', which makes it clear that services should be responsive to patients' needs. Many patients perceive that an integrated specialist clinic would be of benefit to them. ${ }^{5}$

With these issues in mind I recently surveyed the opinion of neurologists throughout the United Kingdom about specialist clinics in general and for Parkinsonism in particular. A questionnaire (Appendix A) was sent to members of the Association of British Neurologists and replies were received from 104 practising neurologists of whom 85 were consultants (that is, approximately $50 \%$ of the UK's consultant neurologists.) The majority of respondents $(67 \%)$ worked at a teaching hospital, $15 \%$ at a District General Hospital and $11 \%$ at both sites.

The majority of respondents $(49 \%)$ thought a clinic for Parkinson's disease would be useful but a third were undecided (table 1 ). Research was cited as the benefit of a clinic by $92(88 \%)$ of respondents, organisation of care by $71(68 \%)$ and teaching by $47(45 \%)$. Interestingly, of the 51 respondents who favoured a Parkinsonism clinic, $90 \%$ of them cited organisation of care as a benefit of the clinic. Other benefits mentioned were greater experience of problems, continuity of care, training of junior staff, the ability to mobilise other resources and patient satisfaction.

Disadvantages of a clinic were given by 87 


\section{Appendix A}

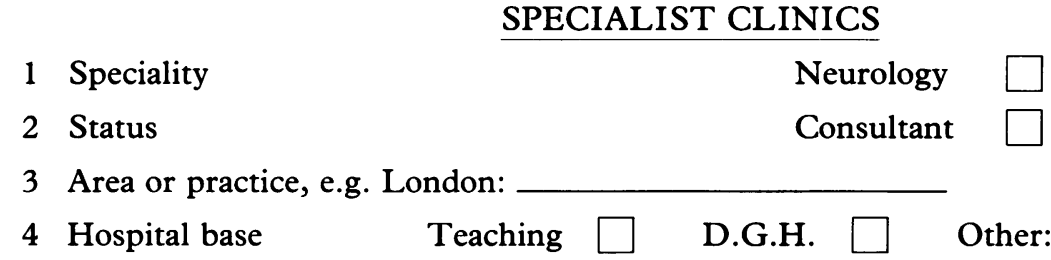

5 Do you feel that specialist clinics can be useful?

6 Do you run a specialist clinic?

Yes $\square=\begin{aligned} & \text { No } \square \\ & \text { Yes } \square\end{aligned}$ No $\square$

7 Which specific interest does this cover?

\section{Epilepsy \\ Stroke disease \\ Parkinsonism}

Other:

8 Do you feel a specialist clinic for Parkinsonism would be useful?

9 What are the benefits?

Organisation of care

Research

Teaching

Other:

10 What are the disadvantages?

Please state:

11 Would you run a Parkinsonism clinic?

Yes

No: Not of benefit to patients

General clinic is adequate

Not enough personnel time

Lack of support staff

Other:

(please state)

Thank you for completing the questionnaire.

$(84 \%)$ respondents (table 2$)$. The main ones were the concern that less severely disabled patients would be distressed by seeing their more severely disabled fellow sufferers. Some felt it would be boring and others too demanding. A lack of therapy support was often mentioned but the major theme was their already heavy workload.

Only $20(19 \%)$ respondents would run a Parkinsonism clinic (table 1). Seventy six $(73 \%)$ respondents gave reasons why they would not run a clinic. These were similar to

Table 1

\begin{tabular}{|c|c|c|c|}
\hline Question & Yes & $\begin{array}{l}\text { Numbers } \\
(\%) \\
\text { No }\end{array}$ & Undecided \\
\hline $\begin{array}{l}\text { Can specialist clinics } \\
\text { be useful? }\end{array}$ & $95(91)$ & $8(8)$ & 1 \\
\hline $\begin{array}{l}\text { Do you run a specialist } \\
\text { clinic? } \\
\text { Would a specialist }\end{array}$ & $52(50)$ & $52(50)$ & \\
\hline $\begin{array}{l}\text { Parkinsonism clinic be } \\
\text { useful? } \\
\text { Would you run a }\end{array}$ & $51(49)$ & $35(34)$ & $18(17)$ \\
\hline Parkinsonism clinic? & $20(19)$ & $76(73)$ & $8(8)$ \\
\hline
\end{tabular}

the disadvantages given in table 3 but also stated that the general clinic was adequate or asked whether a specialist clinic would provide greater benefit for the patients.

Clearly the information from the questionnaire can be biased by the way in which questions are framed. Nonetheless, I think this survey gives some useful insight into the views of neurologists in the UK, in particular their anxieties about specialist clinics, as it is clear that the general concept is acceptable but the practice, certainly for Parkinsonism, is not.

\section{OTHER FACETS OF CARE}

There are, however, other significant concepts, which could be usefully incorporated into any management strategy for chronic neurological disease. Such strategies will of course be influenced by the availability of resources at local level and to some extent by the particular interests of the health care workers.

Patients with Parkinsonism indicate strongly that they wish to be seen by a specialist. It seems less important to them what the specialty 
Table 2 Disadvantages of a specialist clinic ( 87 respondents)

Mix of severe and less disabled

2 Hived off to junior staff

3 Dilution of general experience

Danger of every complaint being attributed to PD Boredom-too demanding

Lack of paramedical support

Workload already too great

Implications for management of other conditions

is (that is, neurologist, geriatrician or rehabilitationist) than that the specialist is interested and has the necessary expertise. This desire, however, has tremendous implications for workload since it has been estimated that in a health district with a population of 250000 people there will be around 400 people with Parkinson's disease ${ }^{6}$ and on average only one neurologist. ${ }^{7}$

\section{SHARED CARE, MANAGEMENT PROTOCOL, REFERRAL CRITERIA}

Structured shared care between the general practitioner and the specialist, as is practised in the managment of diabetes, is the only realistic option which can fulfil patient aspirations. A recent survey of 400 general practitioners throughout the UK gives an idea of their perception of how Parkinson's disease patients should be managed. They were asked to identify how their patients were managed and how they would like them to be managed. Seventy two per cent felt care was provided by the general practitioner, $10 \%$ by the specialist and $18 \%$ by some form of shared care. Only $33 \%$ felt general practitioner care was the ideal, $10 \%$ that specialist care was ideal and $57 \%$ shared care. ${ }^{8}$ These data tend to support the suggestion that, for whatever reason, many patients with Parkinson's disease are not referred for specialist support, ${ }^{9}$ nonetheless it is clear that general practitioners would wish to share the care of these patients. Shared care rightly emphasises that patients continue to live in the community, and the role of the general practitioner is pivotal. Shared care should allow easier and more timely access to specialist expertise and the associated multidisciplinary team. To be effective it should be run in conjunction with a locally agreed management protocol incorporating audit criteria. An integral part of this is agreed referral points, which are not intended to be prescriptive but rather to provide good patient care (table 3 ). Clearly they will depend on the level of experience of the local practitioners and do not preclude referral at other times. They should be known both by patients and carers so that they can seek timely help and be involved in the

Table 3 Possible referral criteria

Confirm diagnosis

Deteriorating function

Marked drug side effects

Swallowing problems

Nightmares/Hallucination

Confusion/Depression

Later motor problems, for example, fluctuations

Considering "drug holiday"

Carers relief

Terminal care monitoring of their condition and the resulting management decisions.

The referral points illustrated represent areas of concern for patients and can constitute major management problems. For example, confirmation of the diagnosis and by implication early referral are important. Patients have particular anxieties at this time and we know from the Romford project the value of special attention to the explaining the diagnosis and providing further information and support at that time. ${ }^{10}$ In addition, initial assessment by a specialist is useful, as patients are often started inappropriately on medication which is potentially toxic for conditions which are not levodopa responsive, such as essential tremor. Even with an "expert" opinion, as many as $15 \%$ of the brains of confidently diagnosed patients had no pathological evidence of Parkinson's disease when examined in the Parkinson's Disease Society Brain Bank.

\section{MULTIDISCIPLINARY TEAM WORK}

Team work and multidisciplinary assessment are the corner stones of effective management for multiple problems such as those faced by Parkinson's disease patients and their carers. Geriatricians have long practised in this way and the value of such an approach for neurological problems, even in the early stages, has recently been demonstrated. ${ }^{10}$ The Government has given its support by highlighting the priority which should be given to multidisciplinary assessment in its White Paper "Caring for People"." The skills of many professionals will be required from time to time but a core team should include the general practitioner, specialist nurse, occupational therapist, speech therapist, physiotherapist, social worker and hospital specialist. For some patients it may be necessary to appoint a key worker who may also act as the patient's advocate. The focus should be on the patient and solving or at least ameliorating their problems rather than the disease process itself and the patient and their carer should be fully participating members and not passive recipients. Whilst they should be involved early in the disease there is no doubt that, at a time when drug treatment begins to fail the patient, the contributions of the team can greatly influence the subsequent quality of life both for the patient and carer.

\section{SPECIALIST NURSE}

The potential contribution of nursing colleagues to the management of chronic neurological disease is often underestimated and underused. The skills of specialist nurses are already used in many areas, such as diabetes, continence, stoma care, infection control, community psychiatric nursing and even intensive care. As well as dealing effectively with many routine management problems they can provide advice and education not only for patients and carers but also fellow professionals. A recent report from the Parkinson Disease Society Nurse Working Party has been very useful in highlighting the problems which those with Parkinson's disease experience on 
admission to hospital, in particular to surgical wards. ${ }^{12}$

\section{ADDRESSING ANXIETIES}

Whilst not essential, I believe these management strategies would be facilitated by an identifiable base such as a specialist clinic. An appropriate model might be the geriatric medicine day hospital ${ }^{13}$ and indeed this is used by many geriatricians who run a Parkinsonism clinic. With this in mind I will discuss the anxieties about such clinics which emerged from my survey (table 3 ).

Patients seem less concerned about mixing with people of differing levels of disability than do their physicians. However, it is not appropriate for the most severely disabled to attend a clinic. They will be better served by a visit in their own homes by their general practitioner or specialist as part of the multidisciplinary team or the specialist nurse, or when appropriate, a particular therapist as an outreach facility linking the hospital and the community. An appointment system could also be used to good effect. Any management protocol should ensure that the clinic is not run by junior staff and time spent with the specialist nurse and the therapists on return visits may often be more profitable.

Staff from other disciplines will have the opportunity to gain concentrated experience and to observe and take part in a centre of excellence and thus further their education. It is my experience that working in a specialist clinic makes you more aware of the dangers of every complaint being attributed to Parkinsonism. In addition, for those who are afraid of boredom this concern should keep them on their toes!

Access to therapy staff is essential for such a clinic but I suspect that management may be more inclined to support a request for a therapist when there is a specific client group with definable problems and the therapist will form part of a specialist team (perhaps with publicity value) rather than a request for a therapist to service the neurology patients in general.

Finally the vexed question of workload. There can be no doubt that, as reported by Hopkins et al, ${ }^{14}$ a median of 79 patient (range 33-144) encounters per week of whom $41 \%$ were new patients, is a considerable workload.

In addition to the contribution of a specialist nurse and the implementation of structured shared care, doctors from other disciplines could help to share the workload in a specialist clinic. It is clear that many neurological problems are dealt with by other specialties as well as by general practitioners and although the issue of quality of care provided by nonneurologists has not been addressed the question has been posed: are all neurological problems the domain only of neurologists? ${ }^{9}$ Pragmatically the answer is no, since geriatricians, for example, find that neurological problems and in particular Parkinsonism are a large part of their daily practice. They also have long established skills in rehabilitation and extensive experience of the multidisciplinary team as a management tool.
It is clear that if neurologists wish to involve themselves routinely in rehabilitation and ongoing care more of them will be required but it is by no means certain that money will be available to fund any such expansion. Also senior registrars will require to be trained in rehabilitation. A short attachment to geriatric medicine would give useful insight into rehabilitation and also expose neurologists in training to the wide spectrum of neurological disease in old age. In the meantime with patients' expectations of care continuing to rise, a more flexible approach such as collaboration of specialists, bringing already established complementary skills to the setting of a specialist clinic, should be examined more closely. Some will say that this already happens with the cross-referral system between specialists but in reality this is often extremely haphazard and the resulting standards of care are by no means universally high.

An attractive alternative to specialist clinics and services is the creation of a generic disability service, ${ }^{15}$ for which it is not necessarily the knowledge and expertise in a particular disease which is relevant but the philosophy and approach to the problems of multiple disability, whatever their cause. The principles of such a service have been documented by Wade.$^{16}$ It would appear to require the creation of a new speciality and the implication of new building or re-allocation of existing beds to accommodate their patients. Specialist clinics simply require a more flexible use of existing clinic or day hospital space and the willingness of doctors to work together. Patients also appear to lay stress on seeing people who have knowledge and expertise in their particular disease. Rightly or wrongly specialist clinics acknowledge that plea but use as their working ethos the same philosophy and approach as a disability clinic and service. I suspect that without a firm government initiative towards disability services the approach adopted will inevitably depend on local factors. With the inception of shared care, management protocols and more informed patients, specialist clinics could become a feasible proposition in terms of medical time and manpower. The clinic as such, however, can only be seen as part of a service which is reflected in the other facets of care I have discussed. The resulting package when combined with provision of respite and terminal care should provide a flexible and effective management tool for Parkinsonism and other chronic neurological conditions.

1 Working for Patients. London: HMSO, 1988.

2 Research Unit, RCP, London. Standards of care for patients with neurological disease. J Roy Coll Phys Lond 1990; with neur

3 Mutch WJ, Strudwick A, Roy SK, Downie AW. Parkinson's disease: disability, review and therapy. $B M J$ 1986; 293:675-7.

4 Lees AJ, Blackburn NA, Campbell VL. The Night-time problems of Parkinson's disease. Clin Neuropharmacol problems of

5 Williams $\mathrm{J}$. Why integrated clinics would improve PD management. Geriatric Medicine 1988;18:30-3.

6 Wade DT, Langton Hewer R. The epidemiology of some neurological diseases. Int Rehabil Med 1987;8:129-37.

7 Langton Hewer R, Wood VA. Report on neurology services in the United Kingdom: 1988 Frenchay Hospital, Bristol. 
8 Mutch WJ. Tried and trusted therapy makes up for lack of GP support Geriatric Medicine 1990;20:67-70.

9 Stevens DL. Neurology in Gloucestershire: the clinical workload of an English neurologist. J Neurol, Neurosurg, Psychiatry 1989:52:439-46.

10 Oxtoby M, Findley L, Porteous A, et al. A strategy for the management of Parkinson's disease and for the long-term support of patients and their carers. London: Parkinson's Disease Society, 1988.

11 Caring for people (community care in the next decade and beyond). London: HMSO, 1989.

12 Report of Nurse Working Party. London: PDS, 1990.

13 Brocklehurst JC. The geriatric day hospital. London: King Edward's Hospital Fund for London, 1979.
14 Hopkins A, Menken M, Defriese G. A Record of patient encounters in neurological practice in the United Kingdom. J Neurol, Neurosurg Psychiatry 1989;52:436-8.

15 Wade DT. Do we really need a specialist stroke service, Geriatric Medicine 1990;20:47-52.

16 Wade DT. Designing district disability services-the Oxford experience. Clinical Rehabilitation 1990;4:147-58.

I am grateful for the help of the consultant neurologists and senior registrars who completed my questionnaire and Mrs Louise Hutton for typing the manuscript. 